Conformational characteristics of the hexanucleoside pentaphosphate AUAUAU: a 2D NMR study at $500 \mathrm{MHz}$

Peter P.Lankhorst, Gijs A.van der Marel, Gerry Wille, Jacques H.van Boom and Cornelis Altona

Gorlaeus Laboratories, State University of Leiden, P.O. Box 9502, 2300 RA Leiden, The Netherlands

Received 14 March 1985; Accepted 10 April 1985

\title{
ABSTRACT
}

All 36 ribose proton resonances and most of the base proton resonances of the hexanucleoside pentaphosphate AUAUAU have been assigned unequivocally using 2D J-resolved spectroscopy, spin echo correlated spectroscopy (SECSY) and 2D NOE spectroscopy (NOESY).

The NMR parameters of AUAUAU are compared with those of smaller fragments that contain methylated adenine bases: m $A U, m^{6} A U m_{5}^{6} A, m_{2}^{6} A U m_{5}^{6} A U$ and m 6 AUm 6 AUm6. Previous studies on this series of compounds have shown that in all these cases purine-pyrimidine-purine sequences prefer to adopt a mixture of states which have as common feature that the interior pyrimidine residues are bulged out, whereas the purine residues stack upon each other.

Chemical shift data, proton-proton coupling constants, as well as the observation of imino-proton resonances for AUAUAU show unambiguously that upon lowering the temperature the high-temperature "bulged out" situation reverts to a normal A-RNA-like double helix.

\section{INTRODUCTION 1,2}

The advent of modern two-dimensional NMR techniques has enabled the assignment of oligonucleotides of ever increasing length. Since 1982 2D techniques ${ }^{3}$ ", such as COSY, SECSY, J-resolved spectroscopy and NOESY are commonly employed to assign the non-exchangeable proton resonances of oligoribonucleotides $^{5-7}$, as well as of oligodeoxyribonucleotides ${ }^{2-14}$, and of a DNA-RNA hybrid"s. In the case of NMR spectra of deoxyribonucleotides complete assignments have been reported up to the octamer level ${ }^{14}$, and a partial assignment (excluding the $\mathrm{H}^{\prime}$ ' and $\mathrm{H}^{2}$ " proton resonances) of a dodecanucleotide has been published 16 . However, in the case of oligoribonucleotides complete assignments are rare, and to the best of our knowledge no such assignments of spectra of hexamers have been reported as yet. Of course, in general the proton spectra displayed by RNA fragments are more complicated than those of DNA oligomers of comparable length. This is due to the fact that $\mathrm{H}^{\prime}$ ' of the former resonates within the same crowded spectral region as $\mathrm{H}^{\prime}, \mathrm{H} 4^{\prime}, \mathrm{H} 5^{\prime}$ and $\mathrm{H}^{\prime \prime}$. With a few exceptions $1,9,13,15$ thus far no sets of coupling constants have been reported in NMR studies of oligonucleotides containing six or more residues. 
From a conformational point of view this situation is disappointing, because coupling constants represent a valuable source of information concerning the geometry of nucleic acid constituents.

In the present paper an NMR study of the RNA hexanucleoside pentaphosphate AUAUAU is presented. This compound is of considerable interest, as it contains two purine-pyrimidine-purine base sequences. Lee and Tinoco'7-19 have suggested from an NMR study on thirteen trinucleoside diphosphates that those three examples with a purine-pyrimidine-purine type of base sequence prefer to adopt a conformation in which the two purines are stacked and the pyrimidine is bulged out of the stack.

In several previous studies from this laboratory the phenomenon of "bulged bases" was investigated thoroughly using the series $\bar{A} \cup \bar{A}^{5}, \bar{A} \cup \bar{A} \cup^{20}$ and $\bar{A} \cup \bar{A} \cup \bar{A}^{7}$ $\left(\bar{A}=m_{2}^{6} A\right)$ as model compounds. $\bar{A} \cup \bar{A}$ represents an example of a trinucleotide with a bulged interior $U$-residue; in $\bar{A} \cup \bar{A} U$ the $\bar{A} \cup \bar{A}$-part has similar characteristics as the trimer, however, the $3^{\prime}$-terminal $\bar{A} U$ moiety is involved in a regular stacking interaction; in the pentanucleotide $\bar{A} \cup \bar{A} \cup \bar{A}$, two bulged $U$-residues exist side by side (Figure 1 ). In contrast to these $\bar{A}$ and $U$ containing oligonucleotides, the hexanucleotide AUAUAU contains non-methylated adenine bases. Therefore, the hexamer in principle is capable of forming a classical Watson-Crick-type RNA duplex.

At this point the question arises whether or not the capability of forming interstrand hydrogen bridges disturbs the usual pattern of bulging $U$ residues. This question is of some importance, as several studies have indicated that bulged residues may occur in double helical RNA fragments when extra non-Watson-Crick bases are inserted into a double helical fragment $\mathbf{2 1}^{222}$. Moreover, single bulged residues frequently occur in natural ribosomal $\mathrm{RNA}^{23,24}$, and it has been suggested that these residues play an important role in the formation of stable 5S RNA-protein complexes ${ }^{25}$.

In the present study the complete assignment of all resonances (except three $\mathrm{H} 2$ resonances of adenine) by means of two-dimensional NMR is described. Moreover, a virtually complete set of ${ }^{3} \mathrm{~J} / \mathrm{HH}$ coupling constants is presented, which enables the determination of the preferred conformations of the sugar rings and the backbone angles $\gamma$.

\section{MATERIALS AND METHODS}

\section{NMR samples}

The title compound was synthesized via an improved phosphotriester approach $^{26}$ and treated with Dowex-cation exchange resin to yield the sodium salt. $16 \mathrm{mg}$ hexanucleotide was lyophilized three times from $99.758 \mathrm{D}_{2} \mathrm{O}$ and taken up 


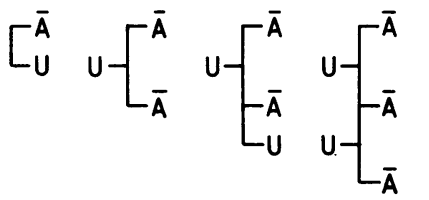

Figure 1: Schematic representation of the preferred conformations of $\bar{A} U, \bar{A} \cup \bar{A}, \bar{A} \cup \bar{A} U$ and $\bar{A} \cup \bar{A} \cup \bar{A}$.

in $0.4 \mathrm{ml} 99.958 \mathrm{D}_{2} \mathrm{O}$; the $\mathrm{pH}$ was adjusted to $7.5 \pm 0.5$ (meter reading); tetramethyl ammonium chloride (TMA) was added as an internal reference and ethylenediaminotetraacetic acid was added to neutralize paramagnetic contaminations. Finally, the sample was degassed and sealed under dry nitrogen gas.

A second sample was prepared from $4 \mathrm{mg}$ of hexanucleotide, which was dissolved in $0.4 \mathrm{ml}$ of a $85 / 15 \mathrm{H}_{2} \mathrm{O} / \mathrm{D}_{2} \mathrm{O}$ mixture in order to observe the imino proton resonances.

NMR spectroscopy

NMR spectra were acquired on a Bruker WM-500 spectrometer, equipped with a probe for triple resonance purposes. 1D spectra were recorded on $8 \mathrm{~K}$ datapoints. Resolution was enhanced by application of a Gaussian window and FIDs were zero-filled to $32 \mathrm{~K}$ before Fourier transformation.

The SECSY spectrum was recorded using the basic pulse sequence of $\mathrm{Na}$ gayama et $\mathrm{al}^{27}$ with the following modifications (eq. 1): the pulse sequence was preceded by a $180^{\circ}$ pulse and an appropriate delay in order to suppress the residual HDO peak ${ }^{28}$. Moreover, the usual second $90^{\circ}$ pulse was replaced by a $45^{\circ}$ pulse in order to obtain a better resolution near $f_{1}=0^{29}$. Simultaneous ${ }^{31} \mathrm{P}$ decoupling greatly improved the resolution of cross-peaks. 256 FIDs consisting of 2K datapoints were recorded, a sine-bell window was applied in both directions. Before Fourier transformation the spectrum was zero-filled to $1 K\left(f_{1}\right)$ and $4 K$ $\left(f_{2}\right)$, respectively.

$$
\left[180^{\circ}-\tau-90^{\circ}-1 / 2 t_{1}-45^{\circ}-1 / 2 t_{1}-t_{2}\right]_{n}
$$

The J-resolved spectrum was recorded using the pulse sequence of Nagayama et $\mathrm{al}^{30}{ }^{\circ}$, with a preceding $180^{\circ}$ pulse and simultaneous ${ }^{31} \mathrm{P}$ decoupling (eq. 2). 64 FIDs consisting of $8 K$ datapoints were recorded. A phase shifted (1/6 $\pi)$ sine-square window was applied in the $f_{1}$ direction and a sine-bell window was used in the $f_{2}$ direction. Before Fourier transformation the spectrum was zero-filled to 256 and $16 \mathrm{~K}$ datapoints, respectively.

$$
\left[180^{\circ}-\tau-90^{\circ}-1 / 2 t_{1}-180^{\circ}-1 / 2 t_{1}-t_{2}\right]_{n}
$$

The above-mentioned $2 \mathrm{D}$ spectra were recorded at $32^{\circ} \mathrm{C}$. This temperature was selected as it provides the most favourable conditions for the complete assign- 


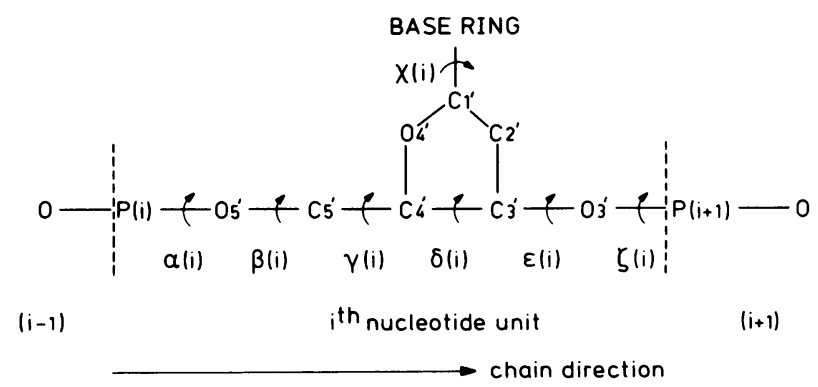

Figure 2: Conformational nomenclature.

ment of the hexamer. At this particular temperature the residual HDO peak resonates within a blank spectral region and at the same time overlapping of high-field ribose signals is reduced to a minimum.

The NOESY ${ }^{31}$ spectrum (eq. 3) was recorded at $27{ }^{\circ} \mathrm{C}$. The residual solvent peak was suppressed by selective saturation of the HDO signal. A mixing time of $0.8 \mathrm{~s}$ was chosen. Although this mixing time certainly does not exclude magnetization transfer by "spin diffusion", and therefore cannot provide reliable distance information, it serves well for assignment purposes in nucleic acid chemistry.

$$
\left[90^{\circ}-t_{1}-90^{\circ}-t_{m}-90^{\circ}-t_{2}\right]_{n}
$$

The imino proton spectra were measured in a $\mathrm{H}_{2} \mathrm{O} / \mathrm{D}_{2} \mathrm{O}(85 / 15 \mathrm{v} / \mathrm{v}) \mathrm{mix}-$ ture. In order to suppress the strong solvent signal, the solvent suppression technique as described by Haasnoot ${ }^{32}$ was used. This technique essentially rests upon the combined use of time-shared long pulses and a data handling technique.

Nomenclature

The proposed IUPAC-IUB nomenclature is used throughout this work. A schematic representation is shown in Figure $2^{33}$.

\section{RESULTS AND DISCUSSION}

\section{Assignment of the sugar and base proton resonances}

The following strategy was adopted for the assignment of the non-exchangeable proton resonances of the hexamer AUAUAU. The projection of the ${ }^{31} \mathrm{P}$ decoupled $2 \mathrm{D} \mathrm{J}$-resolved spectrum recorded at $32{ }^{\circ} \mathrm{C}$ (Figure $3 \mathrm{~A}$ ) yields the accurate peak positions of 44 out of 48 resonances. The remaining 4 resonances cluster at $\sim 1.6 \mathrm{ppm}$ and do not appear as well-resolved singlets, due to strong mutual couplings. Note, that ${ }^{31} \mathrm{P}$ decoupling is essential, because other- 


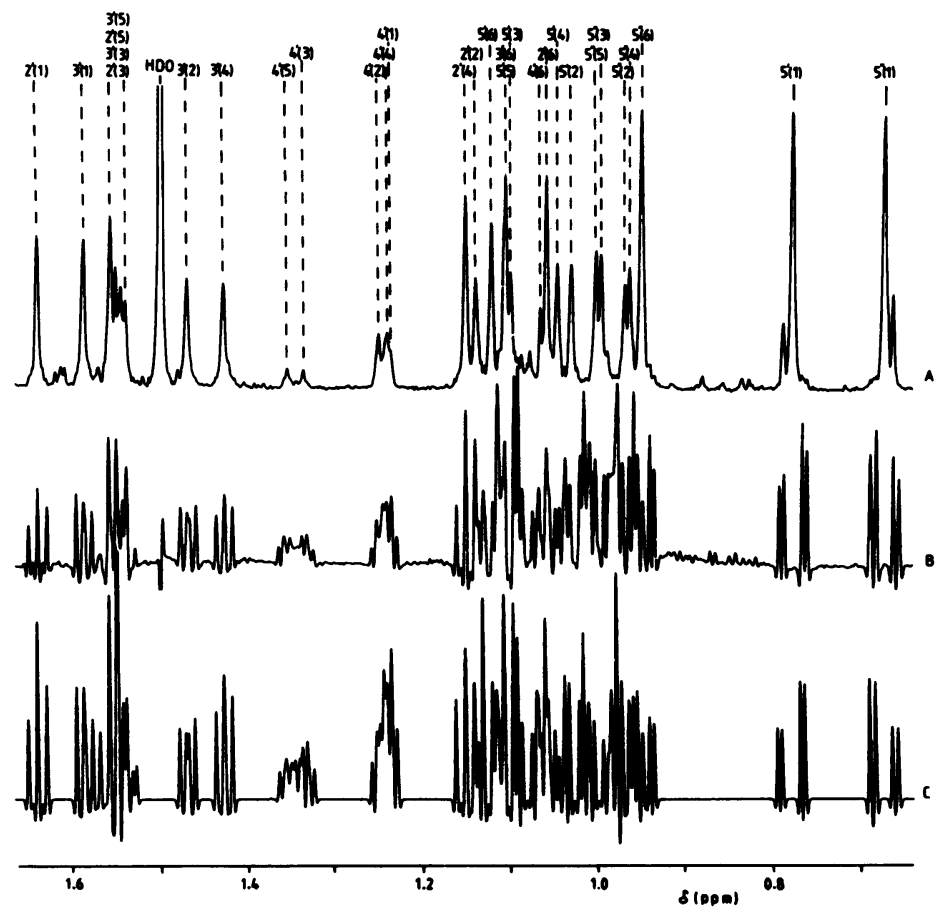

Figure 3: High-field region of ${ }^{2} \mathrm{H}$ spectrum of AUAUAU. A) Projection of the ${ }^{31 \mathrm{P}}$ decoupled $\mathrm{J}$-resolved spectrum. B) One dimensional ${ }^{31 P} \mathrm{P}$ decoupled ${ }^{1} \mathrm{H}$ spectrum. C) Computer simulation of $\mathrm{B}$.

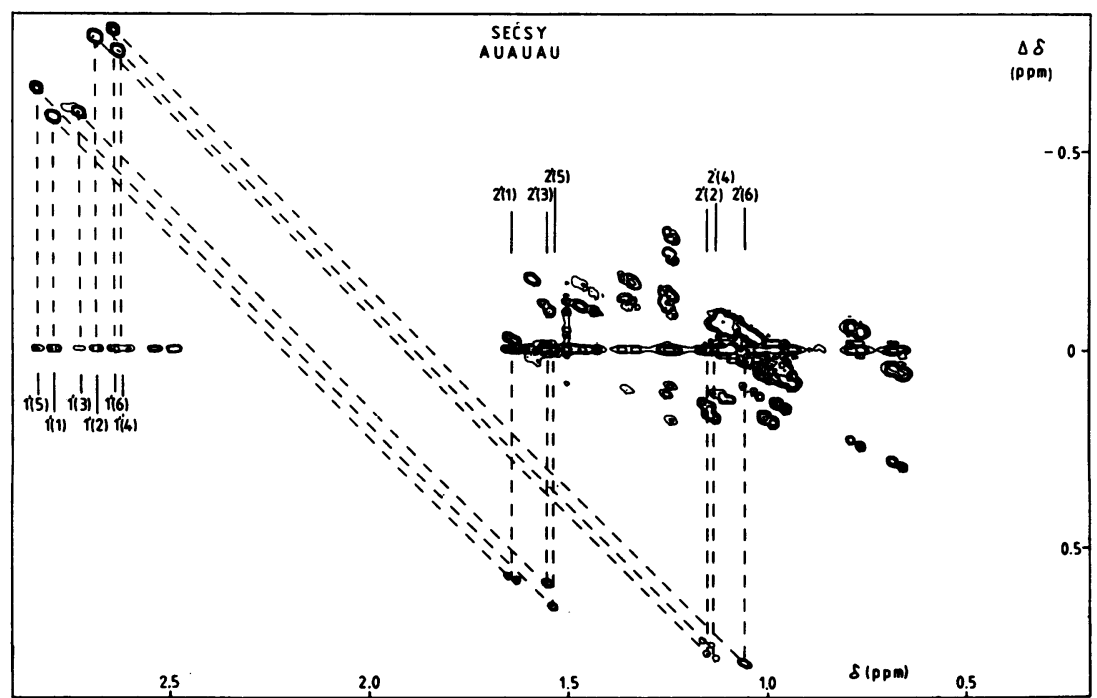

Figure 4: Contour plot of the 31P decoupled SECSY spectrum of AUAUAU. Connectivities between the $\mathrm{H}^{\prime}$ ' and $\mathrm{H}^{\prime}$ ' protons are shown. 


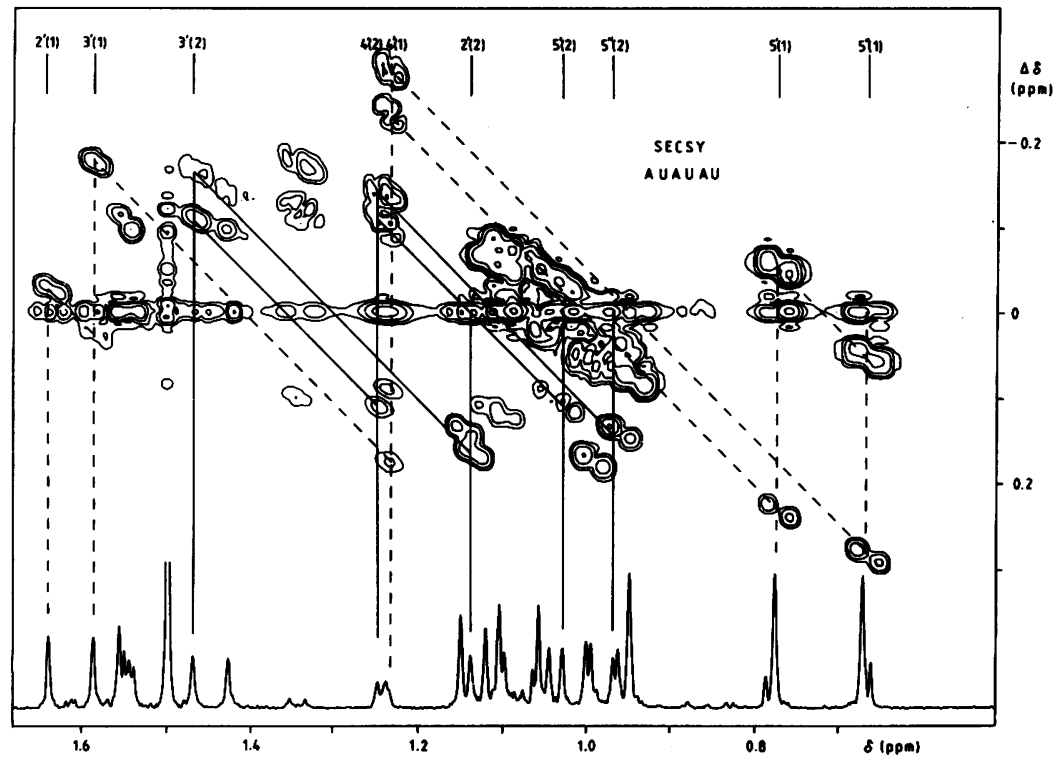

Figure 5: Expanded contour plot of the SECSY spectrum. The high field ribose region is shown, together with the projection of the J-resolved spectrum. Connectivities between the ribose protons of residues 1 and 2 are indicated by a broken line and a solid line, respectively.

wise the projection would consist largely of overlapping doublets, which would effectively block an unambiguous assignment. Through coupling pattern recognition as well as by means of chemical shift considerations, each resonance could be attributed in a straightforward way to a specific type of proton (e.g. H1', H2' etc.). It turned out that the clustering four resonances at $\sim 1.6 \mathrm{ppm}$ arise from two pairs of $\mathrm{H}^{\prime}$ ' and $\mathrm{H}^{\prime}$ ' protons.

The next step of the assignment procedure consisted of the identification of connectivities due to scalar couplings. These connectivities are apparent from the ${ }^{31} \mathrm{P}$ decoupled SECSY spectrum (Figures 4 and 5). Careful inspection of a contour-plot of this spectrum allows the assignment of all sugar protons to six different independent spin systems. Moreover, the H5-H6 connectivities are revealed. However, the SECSY spectrum does not inform us as to which spin system belongs to which nucleotide residue.

The 5'-terminal residue and the 3 '-terminal residue are characterized immediately, because these residues lack a $5^{\prime}$ and a $3^{\prime}$ phosphate group, respectively. This leads to a characteristic high-field shift of the respective H5', H5" and $\mathrm{H}^{\prime}$ resonances, as well as to the absence of the corresponding ${ }^{2} \mathrm{H}-{ }^{3}{ }^{1} \mathrm{P}$ couplings in the ${ }^{32} \mathrm{P}$ coupled spectrum. The remaining residues are assigned from the 


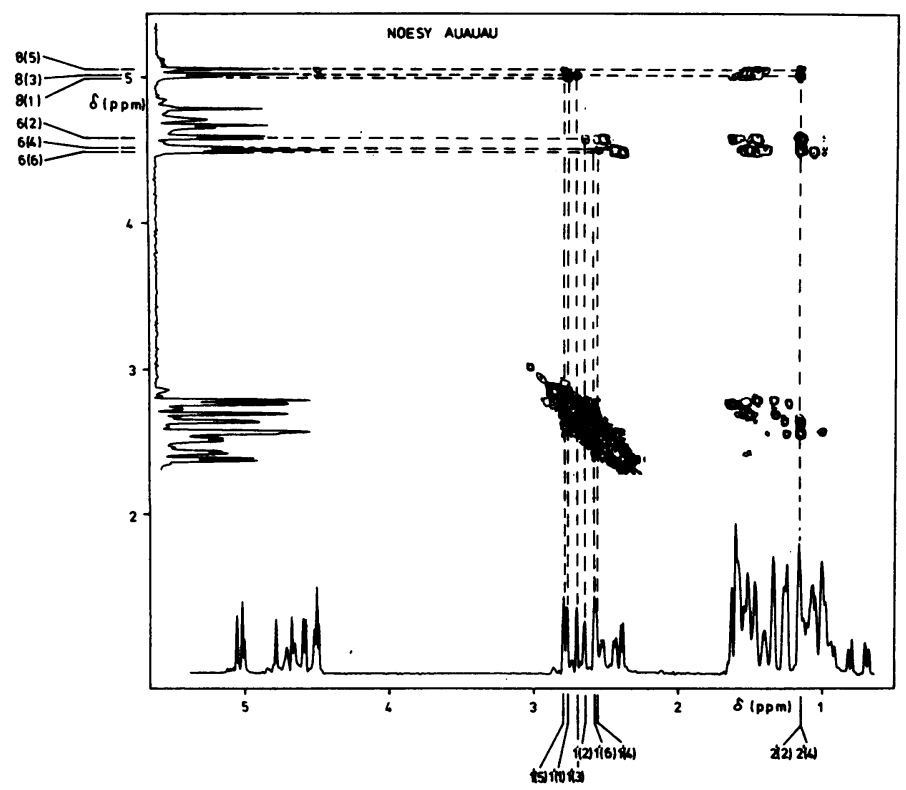

Figure 6: Contour plot of a NOESY spectrum of AUAUAU at $300 \mathrm{~K}$.

NOESY spectrum, (Figure 6).

Three cross peaks are seen which connect adenine $\mathrm{H} 8$ resonances and $\mathrm{H} 1^{\prime}$ resonance positions of the same residue and another set of three cross peaks connect uridine $\mathrm{H} 6$ peaks with the corresponding $\mathrm{HI}^{\prime}$ resonance positions. At this point the six sets of spin systems can be allotted to three that belong to adenine residues and three that correspond to uridine residues. $A(1)$ and $U(6)$ are already assigned from the lack of coupling to ${ }^{32} \mathrm{P}$ as described above. Thus, it remains to discriminate $A(3)$ from $A(5)$ and $U(2)$ from $U(4)$. This is accomplished with the aid of interresidue NOEs. The low-field doublet of the three $\mathrm{H} 6$ resonances shows a strong cross peak to the known $\mathrm{H}^{\prime}(1)$. Therefore this $\mathrm{H} 6$ signal as well as the NOESY-connected ribose signals are assigned to $U(2)$. The remaining set of uridine resonances therefore belongs to $U(4)$.

In principle, it is possible to assign the two A-residues in a similar way, i.e. from the observation of interresidue NOEs between a specific $\mathrm{H} 8$ resonance and the $\mathrm{H}^{\prime}$ ' of the $5^{\prime}$ linked residue. Unfortunately, the $\mathrm{H}^{\prime}(2)$ and the $\mathrm{H} 2^{\prime}(4)$ signals of AUAUAU are isochronous at $27^{\circ} \mathrm{C}$ and cannot be used to discriminate between $H 8(3)$ and $H 8(5)$. Note, however, that the cross peaks in question indeed are present. The $\mathrm{H} 8 / \mathrm{H} 6$ cross peaks are not conclusive either as can be glanced from Figure 6. For these reasons ID NOE difference experiments were 
Table 1: Chemical shifts (ppm) and coupling constants (Hz) of AUAUAU at $32{ }^{\circ} \mathrm{C}$. Chemical shifts are referenced to the methyl peak of tetramethyl ammonium chloride. For conversion to the DSS scale add $+3.18 \mathrm{ppm}$.

\begin{tabular}{|l|c|c|c|c|c|c|}
\hline proton shift & $\mathrm{A}(1)$ & $\mathrm{U}(2)$ & $\mathrm{A}(3)$ & $\mathrm{U}(4)$ & $\mathrm{A}(5)$ & $\mathrm{U}(6)$ \\
\hline H8 & 5.013 & -- & 5.070 & -- & 5.132 & -- \\
H6 & -- & 4.602 & -- & 4.554 & -- & 4.565 \\
H5 & -- & 2.609 & -- & 2.539 & -- & 2.491 \\
H1' & 2.792 & 2.687 & 2.730 & 2.631 & 2.834 & 2.642 \\
H2' & 1.644 & 1.135 & 1.544 & 1.155 & 1.548 & 1.063 \\
H3' & 1.592 & 1.474 & 1.562 & 1.431 & 1.562 & 1.109 \\
$\mathrm{H}^{\prime}$ & 1.243 & 1.254 & 1.339 & 1.244 & 1.357 & 1.069 \\
$\mathrm{H}^{\prime}$ & 0.780 & 1.032 & 1.104 & 1.049 & 1.109 & 1.125 \\
H5' & 0.678 & 0.976 & 1.002 & 0.969 & 1.007 & 0.955 \\
\hline coupling & & & & & & \\
\hline $1^{\prime}{ }^{\prime}{ }^{\prime}$ & & & & & & \\
$2^{\prime} 3^{\prime}$ & 5.5 & 5.9 & 5.1 & 5.8 & 4.2 & 4.1 \\
$3^{\prime} 4^{\prime}$ & 5.1 & 5.2 & 5.5 & 5.1 & 5.5 & 5.4 \\
$4^{\prime} 5^{\prime}$ & 4.0 & 3.7 & 4.4 & 4.2 & 5.3 & 5.5 \\
$4^{\prime} 5^{\prime \prime}$ & 2.5 & 2.5 & 2.6 & 2.5 & 2.6 & 2.7 \\
$5^{\prime} 5^{\prime \prime}$ & 3.3 & 3.0 & 3.8 & 3.0 & 3.8 & 2.7 \\
\hline
\end{tabular}

performed at $32{ }^{\circ} \mathrm{C}$, at which temperature the $\mathrm{H} 2{ }^{\prime}(2)$ and $\mathrm{H}^{\prime}(4)$ resonances are sufficiently separated. Indeed, irradiation of each of the two $\mathrm{H} 8$ resonances in turn yield strong and specific NOEs to the corresponding $\mathrm{H}^{\prime}$ ' resonances of the 5 ' linked $U(2)$ and $U(4)$ residues. This experiment effectively completes the assignment.

In Figure 3C a computer simulation of the ${ }^{31} \mathrm{P}$ decoupled spectrum of AUAUAU at $34{ }^{\circ} \mathrm{C}$ is shown. Chemical shifts and coupling constants, which were acquired from the 2D J-resolved experiment were used (after small adjustments) as input data (Table 1). The agreement between simulation and experiment is seen to be excellent, which supports the correctness of the assignment reported above. No attempt was made to obtain a computer simulation of the phosphorus coupled spectrum, as in this case individual signals are barely resolved. For this reason accurate determination of ${ }^{2} \mathrm{H}^{31} \mathrm{P}$ coupling constants is precluded.

Chemical shifts of the non-exchangeable protons

The temperature dependence of proton chemical shifts constitutes a sensitive probe to monitor conformational equilibria. In a previous paper ${ }^{7}$ the temperature dependency of the chemical shifts of $\bar{A} \cup \bar{A} \cup \bar{A}$ was studied. Chemical shifts of the pentamer were compared with those of the appropriate dimers and monomers. At all temperatures the pentamer displays an excess of shielding of the protons of the $\bar{A}$ residues, and a lack of shielding of the protons of the $U$ residues. It was concluded that the $\bar{A}$ bases exert mutual shielding effects, but do not appear to significantly shield the $U$ residues. In other words, the $\bar{A}$ res- 

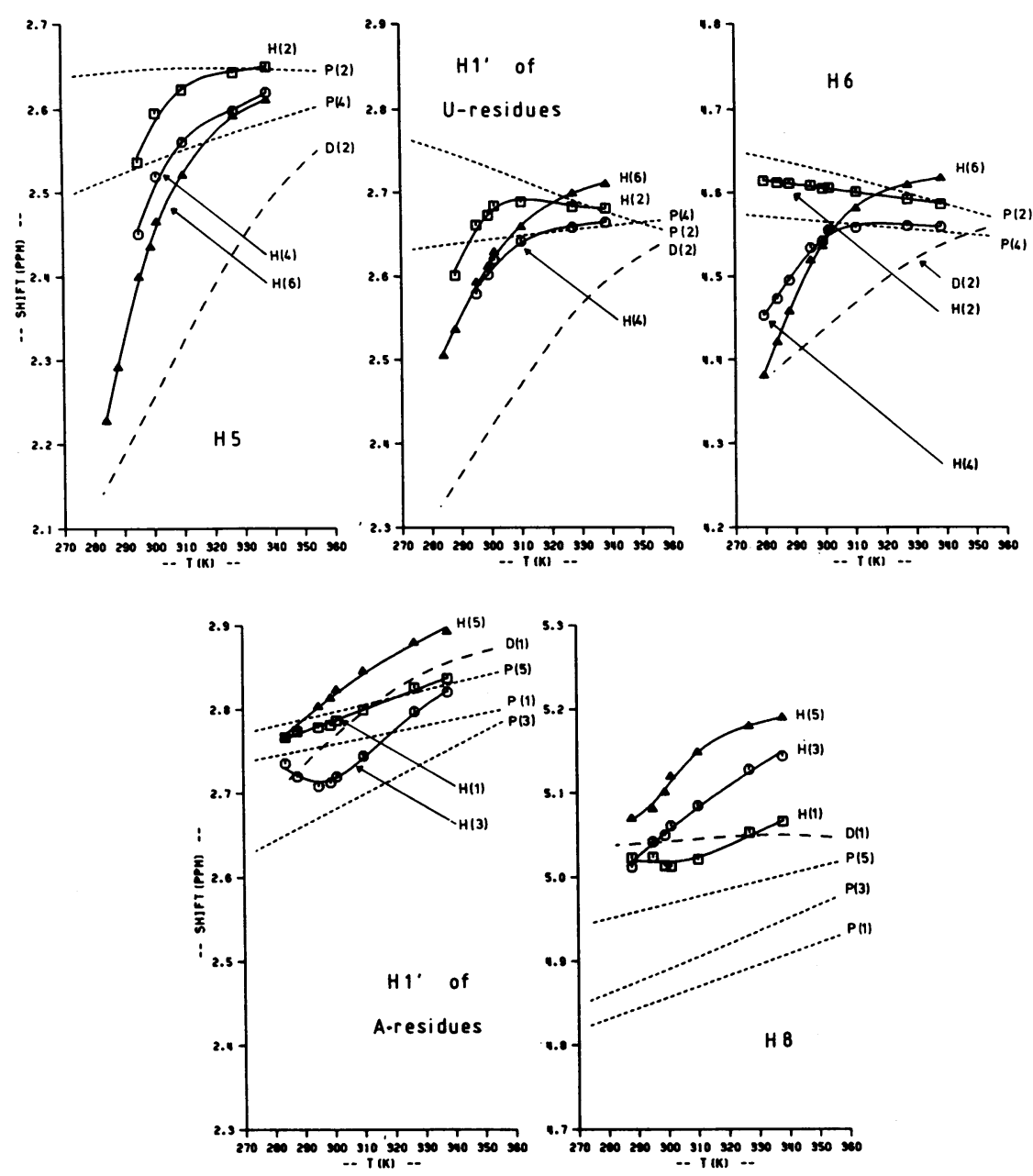

Figure 7: Chemical shifts vs. temperature profiles of $A \cup A \cup A \cup, \bar{A} \cup \bar{A} \cup \bar{A}$ and $\bar{A} U$. From the left to the right(starting at the top) H5, H1' of $U$-residues, H6, H1' of A residues, H8. Symbols $H, P$ and $D$ refer to the hexamer, the pentamer and the dimer, respectively. Thus, for example, $H(3)$ refers to residue 3 of the hexamer.

idues have stacking interactions with each other and the $U$ residues are turned away (bulged out).

In Figure 7 the chemical shift vs. temperature profiles of the 1 ' protons and of the base protons of AUAUAU are compared to those of $\bar{A} \cup \bar{A} \cup \bar{A}$ and to those of $\bar{A} U$. It is apparent from Figure 7 that the three $H 5$ protons of $\bar{A} \cup \bar{A} \cup \bar{A}$, which at elevated temperatures have similar chemical shifts as those of the pen- 


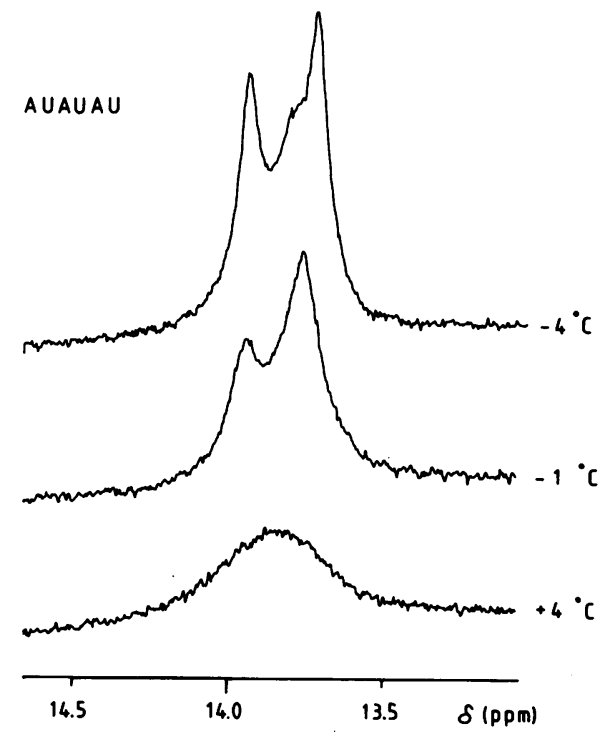

Figure 8: Imino proton spectra of AUAUAU at $-4,-1$ and $+4{ }^{\circ} \mathrm{C}$.

tamer, become strongly shielded when the temperature is lowered. The same effect is seen for the $\mathrm{HI}^{\prime}$ of the residues $U(2), U(4)$ and $U(6)$ and for the $H 6$ of $U(4)$ and $U(6)$. In all these cases the pattern observed for the chemical shift vs. temperature profiles is similar to the behaviour of the $U$ protons of $\bar{A} U$. It is well known that the latter compound displays a strong preference for a stacked state at lower temperatures ${ }^{34}$. It is concluded, therefore, that the $U$ bases of the hexamer at high temperature exist in either a bulge-out situation or in a randomly coiled strand. At temperatures below about $35-40{ }^{\circ} \mathrm{C}$ the $U$ residues become involved in vertical stacking interactions with the $A$ bases. Judging from the relative steepness of the chemical shift vs. temperature profiles compared to those of the dimer $\bar{A} U$, the stacking process in the hexamer is strongly cooperative. This would be expected if the main driving force for this process is the formation of a double helix.

At high temperatures the $\mathrm{H} 8$ protons of the (non-methylated) $\mathrm{A}$ bases in the hexamer resonate 0.15-0.2 ppm downfield from those of the m pentamer. Comparison of the $\mathrm{H} 8$ chemical shifts of non-methylated vs. methylated monomers ${ }^{33}$ shows that N6-dimethylation causes a $0.1 \mathrm{ppm}$ downfield shift of $\mathrm{H} 8$ signals. After correction for the effect of the $\mathrm{CH}_{3}$ groups it is seen that the $\mathrm{H} 8$ protons of the hexamer are slightly deshielded with respect to those of the pentamer at high temperature. Perhaps this is indicative for a weaker A--A next-nearest-neighbour stacking in the non-methylated AUA sequence, analogous to the weaker $A--A$ nearest neighbour stacking in $A p A\left(T_{m} \simeq 295 \mathrm{~K}\right) \mathrm{com}-$ 
pared to $\bar{A} p \bar{A}\left(T_{m} \simeq 318 \mathrm{~K}\right)^{35}$.

At lower temperatures $\left(<35^{\circ} \mathrm{C}\right)$ the chemical shift profiles of the $\mathrm{H} 8$ signals of the hexamer, notably those assigned to $H 8(3)$ and $H 8(5)$, deviate from the $H 8$ profiles of the bulged-out pentamer. In the latter case the signals of $H 8(1)$, $H 8(3)$ and $H 8(5)$ move linearly upfield when the temperature is lowered; in the former case a distinct non-linear upfield shift is seen. Remembering that the chemical shift of $\mathrm{H} 8$ is rather insensitive to the position of the $U$ bases, it is suggested that the $\mathrm{H} 8$ upfield shifts in the hexamer are caused by the ring currents of the $A$ bases in the opposite strand of a double helix which starts to form at low temperatures.

Concomitant with the cooperative shielding effects upon several protons of AUAUAU a gradual broadening of the ${ }^{1} \mathrm{H}$ NMR spectra is seen at temperatures below $30{ }^{\circ} \mathrm{C}$. Below $12{ }^{\circ} \mathrm{C}$ the spectrum rapidly broadens to an extent that all fine structure is lost and no chemical shifts and coupling constants can be measured. This observation forms another strong indication that AUAUAU starts to dimerize into a double helix at lower temperatures. Exchange broadening is commonly observed in a temperature range where a significant population of duplex equilibrates with a significant population of single strands, see for instance reference 36. Unfortunately, the melting temperature of the double helix appears to be rather low $\left(\leq 12^{\circ} \mathrm{C}\right)$. Attempts to reach the plateau region of chemical shifts which correspond to 1008 duplex were unsuccessful. Even when the nucleotide concentration was increased to $60 \mathrm{mM}$ or $1 \mathrm{M}$ salt was added the spectra remained broadened. The rules proposed by Borer et $\mathrm{al}^{\mathbf{3} 7}$ predict a value of $T_{m}$ $\simeq 22{ }^{\circ} \mathrm{C}$ for $20 \mathrm{mM} \mathrm{AUAUAU}$ and $1 \mathrm{M} \mathrm{NaCl}$. The chemical shift vs. temperature profiles obtained in the present study were run in the absence of added salt. Therefore, a verification of this prediction is precluded.

Imino proton spectra of AUAUAU

The imino proton spectra of AUAUAU measured at $-4,-1$ and $+4{ }^{\circ} \mathrm{C}$ are shown in Figure 8. The appearance of three imino proton resonances at 13.7, 13.8 and $14.0 \mathrm{ppm}$ (downfield from DSS) clearly indicates the presence of a double helix with concomitant formation of $A \cdot U$ base pairs. The imino proton peaks broaden out and disappear when the temperature is increased to $+4{ }^{\circ} \mathrm{C}$. The chemical shifts of the imino proton resonances are in good agreement with the values predicted by Patel et al. ${ }^{38}$ (14.0-14.6 ppm).

\section{Conformational analysis of the sugar ring}

The ribose ring can assume either of two geometrical types, the $\mathrm{N}$ conformer or the $S$ conformer ${ }^{9-41}$. It is well known that the ribose rings in A-RNA double helices prefer to assume the $\mathrm{N}$-type conformation, whereas in random 
Table 2: Pseudorotational analysis of the six ribose rings of AUAUAU at $32{ }^{\circ} \mathrm{C}$; an asterisk indicates a constrained value, see text.

\begin{tabular}{|l|l|l|l|l|l|l|}
\hline residue & $\phi(\mathrm{N})$ & $\mathrm{P}(\mathrm{N})$ & $\phi(\mathrm{S})$ & $\mathrm{P}(\mathrm{S})$ & $\mathrm{x}(\mathrm{N})$ & rms $(\mathrm{Hz})$ \\
\hline $\mathrm{A}(1)$ & $37 *$ & 17 & $37 *$ & 158 & 35 & 0.011 \\
$\mathrm{U}(2)$ & $37 *$ & 19 & $37 *$ & 155 & 30 & 0.011 \\
$\mathrm{~A}(3)$ & $37 *$ & 20 & $37 *$ & 152 & 40 & 0.175 \\
$\mathrm{U}(4)$ & $37 *$ & 20 & $37 *$ & 152 & 34 & 0.152 \\
$\mathrm{~A}(5)$ & $37 *$ & 20 & $37 *$ & 153 & 53 & 0.216 \\
$\mathrm{U}(6)$ & $37 *$ & 18 & $37 *$ & $154 *$ & 55 & 0.083 \\
\hline
\end{tabular}

coils an equilibrium between the above-mentioned two conformers exists. Populations of the $N$ and $S$ conformers can be calculated from the vicinal coupling constants by means of computer program PSEUROT 42 . In this approach a two-state equilibrium is assumed, and coupling constants of the ribose ring are translated into torsion angles by means of a generalized Karplus equation ${ }^{4}$, which takes into account the electronegativity and orientation of substituents. The calculated $\phi_{\mathrm{HH}}$ torsion angles are directly related to the pseudorotational parameters of the sugar ring through empirical rules, proposed by de Leeuw et al. ${ }^{44}$.

It was shown earlier ${ }^{5}$ that a full pseudorotational analysis in terms of all relevant parameters is only possible in those cases where several sets of coupling constants have been measured at different temperatures, covering a reasonably large shift of the $\mathrm{N} / \mathrm{S}$ equilibrium constant. In the present case no such data sets are available. Therefore, the amplitudes of puckering $\phi_{N}$ and $\phi_{S}$ were constrained to adopt a value of $37^{\circ}$, which corresponds to the mean value obtained earlier for a number of small RNA fragments"1. Table 2 shows the phase angles of pseudorotation $P_{N}$ and $P_{S}$ and the relative population of $N$ conformer for each of the six ribose rings at $32{ }^{\circ} \mathrm{C}$.

The calculated phase angles are found within the ranges long known from $X$-ray crystallography ${ }^{44}$ and deserve little comment. Of more interest are the relative $\mathrm{N}$ and $\mathrm{S}$ populations. At a temperature of $32^{\circ} \mathrm{C}$, where the population of the duplexed structure is still quite small according to the chemical shift vs. temperature profiles (vide supra), the ribose rings of the hexamer display considerable conformational freedom: 30-40 \& $\mathrm{N}$-type rings being indicated for residues (1) to (4) and about 558 for residues $A(5)$ and $U(6)$. The higher $N$ population for the terminal sequence is not unexpected, because a similar situation occurs in the tetramer $\bar{A} \cup \bar{A} U^{20}$. In the tetramer the 3' terminal $\bar{A} U$ sequence experiences normal base-base stacking with the sugars adopting an $\mathrm{N}$-type conformation when the temperature is lowered, whereas the sugars $\bar{A}(1)$ and $U(2)$ retain conformational flexibility. The present data suggest that some 
Table 3: The population of $\mathrm{N}$-conformer as a function of temperature for AUAUAU, $\bar{A} U \bar{U} U \bar{A}$ and for $\bar{A} U$.

\begin{tabular}{|l|c|c|c|c|c|c|c|c|c|c|c|c|c|}
\hline & \multicolumn{5}{|c|}{ AUAUAU } & \multicolumn{4}{c|}{ AUAUA } & \multicolumn{3}{c|}{ AU } \\
\hline $\mathrm{T}\left({ }^{\circ} \mathrm{C}\right)$ & $\mathrm{A}(1)$ & $\mathrm{U}(2)$ & $\mathrm{A}(3)$ & $\mathrm{U}(4)$ & $\mathrm{A}(5)$ & $\mathrm{U}(6)$ & $\mathrm{A}(1)$ & $\mathrm{U}(2)$ & $\bar{A}(3)$ & $\mathrm{U}(4)$ & $\mathrm{A}(5)$ & $\bar{A}(1)$ & $\mathrm{U}(2)$ \\
\hline 11 & -- & -- & 75 & -- & 80 & 66 & 30 & 25 & 43 & 40 & 52 & 81 & 82 \\
15 & -- & 49 & 66 & -- & 80 & 65 & 30 & 25 & -- & -- & 51 & 79 & 80 \\
22 & 45 & 38 & 54 & 52 & 66 & 62 & 30 & 25 & 41 & -- & 51 & 73 & 76 \\
32 & 35 & 30 & 40 & 34 & 53 & 55 & 30 & 25 & 40 & 41 & 48 & 66 & 72 \\
54 & 33 & -- & 43 & 31 & 49 & -- & 30 & 25 & 38 & -- & 46 & 52 & 65 \\
65 & 32 & 28 & 43 & -- & 48 & 55 & 30 & -- & 38 & -- & 46 & 47 & 62 \\
\hline
\end{tabular}

stacking between $A(5)$ and $U(6)$ also occurs in the hexamer at $32{ }^{\circ} \mathrm{C}$ and only a little between the remaining residues at this temperature.

At this point it becomes mandatory to study the conformational behaviour of the six riboses in the hexamer as a function of temperature. If the present interpretation of the chemical shift profiles in terms of an increasing population of duplex structure at low temperature is correct, then one must expect a concomitant increase of $\mathrm{N}$-type population. This is indeed found. A series of 1D spectra taken at temperatures varying from $11{ }^{\circ} \mathrm{C}$ to $65{ }^{\circ} \mathrm{C}$, were analyzed to extract first order $\mathrm{J}_{1}{ }^{\prime}$ ' couplings. Using the geometrical parameters shown in Table 2 the mole fractions $\mathrm{N}$-type conformer can be reliably approximated from knowledge of $\mathrm{J}_{12}{ }^{\prime}$. The mole fractions of $\mathrm{N}$ conformer are shown in Table 3 , along with the comparable values for $\bar{A} \cup \bar{A} \cup \bar{A}$ and of $\bar{A} U$. Striking similarities and differences between the hexamer and the pentamer are immediately apparent. At elevated temperatures, the hexamer displays a similar preference for $S$-type ribose rings as does the pentamer. Upon lowering the temperature, however, the individual ribose equilibria of the pentamer do not shift at all, whereas the riboses of the hexamer in the interval $32-11{ }^{\circ} \mathrm{C}$ adopt significant populations of the $\mathrm{N}$-type conformation. Of special interest is the $A(5)$ residue which more strongly resembles the behaviour of $\overline{\mathrm{A}} U$.

Table 4: Population distribution of the backbone angle $\gamma$ of AUAUAU (32 ${ }^{\circ} \mathrm{C}$ ), of $\bar{A} U \bar{U} U \bar{A}$ and of $\overline{\mathrm{AU}}\left(43^{\circ} \mathrm{C}\right)$.

\begin{tabular}{|c|c|c|c|c|c|c|c|c|c|}
\hline \multirow[t]{2}{*}{ res. } & \multicolumn{3}{|c|}{ AUAUAU } & \multicolumn{3}{|c|}{ ĀUĀUA } & \multicolumn{3}{|c|}{$\overline{\mathrm{A}} \mathrm{U}$} \\
\hline & $8^{+}$ & $t$ & $8^{-}$ & $8^{+}$ & $t$ & $8^{-}$ & $g^{+}$ & $t$ & $8^{-}$ \\
\hline $\begin{array}{l}A(1) \\
U(2) \\
A(3) \\
U(4) \\
A(5) \\
U(6)\end{array}$ & $\begin{array}{l}78 \\
81 \\
72 \\
81 \\
72 \\
83\end{array}$ & $\begin{array}{l}22 \\
18 \\
27 \\
18 \\
27 \\
18\end{array}$ & $\begin{array}{l}0 \\
0 \\
1 \\
0 \\
1 \\
3\end{array}$ & $\begin{array}{l}76 \\
78 \\
63 \\
77 \\
71 \\
--\end{array}$ & $\begin{array}{l}21 \\
20 \\
33 \\
19 \\
24 \\
--\end{array}$ & $\begin{array}{r}3 \\
2 \\
4 \\
5 \\
6 \\
--\end{array}$ & $\begin{array}{l}76 \\
82 \\
-- \\
-- \\
-- \\
--\end{array}$ & $\begin{array}{l}19 \\
17 \\
-- \\
-- \\
-- \\
--\end{array}$ & $\begin{array}{l}5 \\
1 \\
-- \\
-- \\
-- \\
--\end{array}$ \\
\hline
\end{tabular}


Unfortunately, not all $\mathrm{J}_{1} 2^{\prime}$, couplings could be followed down to $11^{\circ} \mathrm{C}$, but there is no doubt that each individual ribose in the strand follows the same trend. Again, these results point towards the formation of a stacked structure, i.e. a duplex, at low temperature.

Backbone angle O5'-C5'-C4'-C3' ( $\gamma$ )

The conformational situation around $\gamma$ can be monitored by means of $J\left(4^{\prime} 5^{\prime}\right)$ and $J\left(4^{\prime} 5^{\prime \prime}\right)$. The existence of a blend of $\gamma^{t}, \gamma^{-}$and $\gamma^{+}$rotamers is assumed, and the population distribution between these conformers is calculated according to Haasnoot et al." . The results are compared to those obtained earlier for $\bar{A} \cup \bar{A} \cup \bar{A}$ and $\bar{A} U$ in Table 4. Note that the population distributions of the hexamer are given at a slightly lower temperature than those of the pentamer and the dimer. It can be seen that no strong differences between the hexamer and the pentamer occur. This is true because at $32{ }^{\circ} \mathrm{C}$ the population of AUAUAU in the duplex state is still rather small

\section{CONCLUSIONS}

From a number of previous studies $5,7,20$ on RNA fragments containing $\bar{A}$ and $U$ bases it is known that oligonucleotides consisting of purine-pyrimidine-purine base sequences prefer to occur in a mixture of conformational states, which have the following common feature: the central pyrimidine residue in these sequences is not involved in vertical base-base stacking, whereas the two flanking $\bar{A}$ bases exhibit some kind of a stacking interaction (Figure 1). Evidence for this so-called "bulge-out" situation stems in the first place from the absence of shielding effects on the chemical shifts of the protons of the central pyrimidine residue, concomitant with an excess of shielding for the protons of the purine residues. In the second place, the absence of regular right-handed stacking in purine-pyrimidine-purine sequences is clearly evidenced by the larger degree of conformational freedom of the backbone torsion angles $\beta, \gamma$ and $\varepsilon$ as well as by the extraordinarily low populations of $N$ conformer exhibited by the sugar rings (25-50\%).

The abovementioned $\bar{A}$ bases, by virtue of the N6 methylation, are not capable of forming hydrogen bonds to $U$ bases located in an opposite strand. Therefore, all oligonucleotides of this series studied thus far necessarily occur as single-stranded species. Depending on the particular base sequence these single strands may occur as highly stacked species $\left(\bar{A} U^{34}, \bar{A} \bar{A}^{35}, U \bar{A} U^{36}\right.$ and the 3'-terminal moiety of $\bar{A} \cup \bar{A} U^{20}{ }^{\circ}$ ), or as bulged out species ( $\bar{A} \cup \bar{A}^{5}$, the 5 ' terminus of $\bar{A} \cup \bar{A} U^{20}$ and both moieties of $\bar{A} \cup \bar{A} \cup \bar{A}^{7}$ ).

The present study supplies an answer to the question whether or not the bulged-out situation reverts to a helical situation in the case of non-methylated 
self-complementary $[A-U]_{n}$ sequences. Indeed, a number of observations made on AUAUAU provides strong evidence for the existence of an A-RNA-like double helix at low temperature.

1. NMR spectra gradually broaden out below $30^{\circ} \mathrm{C}$.

2. Chemical shifts of the protons of the $U$-residues of AUAUAU diverge from those of $\bar{A} \cup \bar{A} \cup \bar{A}$ below $30^{\circ} \mathrm{C}$, i.e. the former become more shielded.

3. At $-4{ }^{\circ} \mathrm{C}$ three imino proton resonances are seen at $\sim 14 \mathrm{ppm}$ downfield from DSS.

4. All ribose rings of $A \cup A \cup A U$ display a significant and increasing preference for the $\mathrm{N}$ conformer upon lowering the temperature below $30^{\circ} \mathrm{C}$.

Thus, it is concluded that the bulged-out situation, which occurs in purine-pyrimidine-purine base sequences, reverts to a helical state in the case of a self-complementary base sequence, which is capable of forming hydrogen bonds to the opposite strand. Therefore, the formation of an extrahelical base in double-stranded RNA appears to be limited to those cases where an extra residue is inserted within a self-complementary strand.

\section{ACKNOWLEDGEMENTS}

This research was supported by the Netherlands Foundation for Chemical Research (S.O.N.) with financial aid from the Netherlands Organization for the Advancement of Pure Research (Z.W.O.).

Spectra were recorded at the Dutch National 500-200 MHz NMR Facility at Nijmegen and on the $300 \mathrm{MHz}$ spectrometer in the Department of Chemistry at Leiden. We wish to thank Ing. P.A.W. van Dael, Ing. W. Guijt and Drs. C. Erkelens for technical assistance. The help of Dr. C.A.G. Haasnoot and Dr. J.-R. Mellema is gratefully acknowledged.

\section{REFERENCES}

1. This paper is part 41 in the series "Nucleic Acid Constituents" from this laboratory. For part 40 see Lankhorst, P.P., Haasnoot, C.A.G., Erkelens, C., Westerink, H.P., van der Marel, G.A., van Boom, J.H. and Altona, C. (1985) Nucleic Acids Res. in the press.

2. Some oligonucleotides mentioned in this study have modified A-bases: 6-N-(dimethyl)adenine, these will in the remainder of this work be abbreviated as $\bar{A}$. Thus, for example, $6-N-\left(\right.$ dimethyl) adenylyl- $\left(3^{\prime}-5^{\prime}\right)$-uridine is abbreviated as $\bar{A} U$.

3. Benn, R. and Giunther, H. (1983), Angew. Chem. Int. Ed. Engl. 22, 350-380.

4. Wider, G., Macura, S., Kumar, A., Ernst, R.R. and Wuithrich, K. (1984), J. Magn. Res. 56, 207-234.

5. Lankhorst, P.P., Groeneveld, C.M., Wille, G., van Boom, J.H., Altona, C. and Haasnoot, C.A.G. (1982) Recl. Trav. Chim. Pays-Bas 101, 253-263.

6. Westerink, H.P., van der Marel, G.A., van Boom, J.H. and Haasnoot, 
C.A.G. (1984), Nucleic Acids Res. 12, 4323-4338.

7. Lankhorst, P.P., Wille, G., van Boom, J.H. Altona, C. and Haasnoot, C.A.G. (1983), Nucleic Acids Res. 11, 2839-2856.

8. Cheng, D.M., Kan, L.-S., Frechet, D., Ts'O, P.O.P., Uesugi, S. Shida, T. and Ikehara, M. (1984), Biopolymers 23, 775-795.

9. Mellema, J.-R., Pieters, J.M.L., van der Marel, G.A., van Boom, J.H. Haasnoot, C.A.G. and Altona, C. (1984) Eur. J. Biochem. 143, 285-301.

10. Feigon, J., Wang, A.H.-J., van der Marel, G.A., van Boom, J.H. and Rich, A. (1984), Nucleic Acids Res. 12, 1243-1263.

11. Feigon, J., Denny, W.A., Leupin, W, and Kearns, D.R. (1983), Biochemistry 22, 5930-5942.

12. Feigon, J., Leupin, W., Denny, W.A. and Kearns, D.R. (1983), Biochemistry 22, 5943-5951.

13. Lown, J.W., Hanstock, C.C., Bleackley, R.C., Imbach, J.-L., Rayner, B. and Vasseur, J.J. (1984) Nucleic Acids Res. 12, 2519-2533.

14. Broido, M.S., Zon, G. and James, T.L. (1984), Biochem. Biophys. Res. Commun 119, 663-670.

15. Haasnoot, C.A.G., Westerink, H.P., van der Marel, G.A. and van Boom, J.H. (1984), J. Biomol. Struct. Dyns. 1, 131-149.

16. Hare, D., Wemmer, D.E., Chou, S.-H., Drobny, G. and Reid, B. (1983), J. Mol. Biol. 171, 319-336.

17. Lee, C-H and Tinoco, I. (1980) Biophys. Chem. 11, 283-294.

18. Lee, C. -H. (1983), Eur. J. Biochem. 137, 347-356.

19. Lee, C.-H. (1983), Eur. J. Biochem. 137, 357-363.

20. Hartel, A.J., Wille, G., van Boom, J.H. and Altona, C. (1981) Nucleic Acids Res. 9, 1405-1423.

21. Lomant, A.J. and Fresco, J.R. (1972) Prog. Nucl. Acids Res. Mol. Biol. 12, 1-27.

22. Lomant, A.J. and Fresco, J.R. (1973) J. Mol. Biol. 77, 345-354.

23. Woese, C.R., Gutell, R., Gupta, R. and Noller, H.F. (1983) Microbiol. Rev. 47 621-669.

24. Noller, H.F. (1984) Ann. Rev. Biochem. 53, 119-162.

25. Peattie, D.A., Douthwaite, S., Garrett, R.A. and Noller, H.F. (1981) Proc. Natl. Acad. Sci. USA 78, 7331-7335.

26. van der Marel, G.A., van Boeckel, C.A.A., Wille, G. and van Boom, J.H. (1981) Tetrahedron Letters 22, 3887-3890.

27. Nagayama, K., Wuthrich, K. and Ernst, R.R., (1979) Biochem. Biophys. Res. Commun. 90, 305-311.

28. Ohuchi, M., Hosono, M., Matushita, K. and Imanari, M. (1981), J. Magn. Reson. 43, 499-501.

29. Bax, A. and Freeman, R. (1981) J. Magn. Res. 44, 542-561.

30. Nagayama, K., Withrich, K., and Ernst, R.R. (1977) Biochem. Biophys. Res. Commun. 78, 99-105.

31. Macura, S. and Ernst, R.R. (1980), Mol. Phys. 41, 95-117.

32. Haasnoot, C.A.G. and Hilbers, C.W. (1983), Biopolymers 22, 1259-1266.

33. IUPAC-IUB Nomenclature Commission (1993) Eur. J. Biochem. 131, 9-15.

34. Hartel, A.J., Lankhorst, P.P. and Altona, C. (1982) Eur. J. Biochem. 129, 343-357.

35. Olsthoorn, C.S.M., Doornbos, J., de Leeuw, H.P.M. and Altona, C. (1982), Eur. J. Biochem. 125, 367-382.

36. Tran-Dinh, S., Neumann, J.M., Huynh-Dinh, T., Renous, S., Genissel, B., Igolen, J. (1983) Eur. J. Biochem. 133, 579-589.

37. Borer, P.N., Dengler, B., Tinoco, I. and Uhlenbeck, O.C. (1974), J. Mol. Biol. 86, 843-853.

38. Patel, D.J. and Tonelli, A.E. (1974), Biopolymers 13, 1943-1964.

39. Altona, C. and Sundaralingam, M. (1972) J. Am. Chem. Soc. 94, 8205-8212. 
Nucleic Acids Research

40. Altona, C. and Sundaralingam, M. (1973) J. Am. Chem. Soc. 95, 2333-2344.

41. Altona, C. (1982) Recl. Trav. Chim. Pays-Bas 101, 413-433.

42. de Leeuw, F.A.A.M. and Altona, C. (1983) J. Comp. Chem. 4, 428-437.

43. Haasnoot, C.A.G., de Leeuw, F.A.A.M. and Altona, C. (1980) Tetrahedron 36, 2783-2792.

44. de Leeuw, H.P.M., Haasnoot, C.A.G. and Altona, C. (1980) Isr. J. Chem. 20, 108-126.

45. Haasnoot, C.A.G., de Leeuw, F.A.A.M., de Leeuw, H.P.M. and Altona, C. (1979) Recl. Trav. Chim. Pays-Bas 98, 576-577.

46. Hartel, A.J., Gijsman, P. and Altona, C. unpublished results. 